\title{
Influence of initial defects on defect formation process in ion doped silicon
}

\author{
V.A. Smyntyna, O.V. Sviridova \\ I.I. Mechnikov Odessa National University, Physics Department \\ 42, Paster str., Odessa 65023, Ukraine \\ E-mail: sviridova_olya@mail.ru
}

\begin{abstract}
We study the influence of initial defects in high-resistance epitaxial silicon layers of high-resistance epitaxial silicon structures on defect formation processes at ion boron doping. The method of reverse voltage-capacitance characteristics revealed two maxima of dopant concentration in epitaxial silicon layers ion-doped by boron. Studing the structure of the near-surface area in ion-doped epitaxial silicon by means of modern methods has shown that in the field of the first concentration maximum (the nearest one to a wafer surface), the fine-blocked silicon structure is localised. In the range of the second doping concentration maximum, the grid of dislocations with the variable period within one grid and consisting of $60^{\circ}$ dislocations is found out. In the area of dislocation grids, oxygen atoms have been found out. The variable period in the grid is related with a change of mechanical stress and deformation distribution law in the plane of dopant diffusion front as dependent on the presence of initial defects in silicon.
\end{abstract}

Keywords: ion doping, relative deformation, dislocations.

Manuscript received 30.12.08; accepted for publication 18.03.09; published online 20.03.09.

\section{Introduction}

Incentive motive for carrying out these researches is the experimentally established fact that defects at ion doping of silicon are generated even at small currents and doses of a dopant (to $10^{14} \mathrm{~cm}^{-2}$ ) [1-5].

Presence of dislocations in initial epitaxial and monocrystalline silicon samples and heterogeneity of their distribution are caused by a number of well-known factors which can be present both simultaneously and in various combinations. These factors are: thermomechanical stresses, condensation of point defects and impurity segregation. Despite constant improvement of silicon wafer manufacture, these reasons cannot be completely eliminated [1].

Ion implantation is one of the most widespread methods for local silicon doping. The popularity of this method is related with the fact that dopant predepositing is usually carried out at room temperature and provides a high accuracy of doping level control. Further, the temperature drive-in follows (to $900{ }^{\circ} \mathrm{C}$ ), which is necessary for disordered silicon layer crystallization in the region of the first dopant concentration maximum and for creation of uniform dopant concentration distribution in silicon bulk. However, this doping method has certain lacks connected with difficulties of reception a uniform depth profile of doped wafers $[3,4,6]$. These difficulties are caused by generation of defects in the course of the ion implantation process as a consequence of dopant ion interaction with atoms in the lattice sites of the crystal [7] and with initial defects. The existence of local mechanical stresses that lead to local deformations of crystal lattice and influence the plastic flow threshold in semiconductors $[8,9]$ is caused by the presence of initial crystal defects. The mechanism of initial defects influence on the plastic flow threshold is not sufficiently understood. In particular, the mechanism of uncontrollable impurity atoms (oxygen atoms) influence on a level of mechanical stresses localised around dislocations is not ascertained.

Ascertaining the mechanism of the initial defect influence in high-resistance epitaxial silicon structure (HRESS) layers on defect formation process at ion boron doping of these structures is the purpose of this work.

\section{Studied samples and methods of research}

We studied high-resistance silicon structures consisting of low-resistance $n$-silicon substrate and high-resistance epitaxial $i$-silicon layer, i.e. before doping, HRESS 
represented epitaxial $i$ - $n$-structures, where $n$ is a substrate with $\rho=4.5 \mathrm{Ohm} \cdot \mathrm{cm}$, and $i$ is a layer with $\rho=1000 \mathrm{Ohm} \cdot \mathrm{cm}$.

The following methods and equipment were used for researching the silicon wafers:

- method of selective chemical etching by Sirtle [10];

- scanning electron microscopy of surface (SEMS), by means of scanning electron microscopeanalyzer "Cam Scan-4D" with a system of the energetic dispersive analyzer "Link-860" (with the usage of "Zaf" program, mass sensitivity of the device is $0.01 \%$, beam diameter ranges from $5 \cdot 10^{-9}$ to $1 \cdot 10^{-6}$ ) [11], and also by means of scanning electron microscope "PЭМ-106M" manufactured by SELMI;

- optical methods of researches with the usage of metallographical microscope "MMP-2P";

- voltage-capacitance automatic curve tracer with computer processing of information connected to a tablet curve-drawing instrument;

- atomic force microscopy of surface, atomic force microscope (AFM) "NT-206" with the lateral resolution (in the XY plane) of $2-5 \mathrm{~nm}$, and with the vertical resolution (along $Z$ direction) of $0.2-0.5 \mathrm{~nm}$ (depending on rigidity of sample surface);

- setup for ion doping made under the specially developed scheme (Fig. 1).

The technique of experiments consisted in the following. The selective chemical etching method allows one to carry out the level-by-level analysis of silicon by layers etching and to reveal defects simultaneously. After selective chemical etching application, the surface of wafers was investigated on deficiency with the usage of metallographical optical microscope "MMP-2P". To improve the defects image quality and to obtain the possibility of making the quantitative analysis of impurity, we applied such methods of analysis as scanning electron microscopy (using "Cam Scan" and "РЭМ-106М") and atomic force microscopy (using AFM "NT-206") with energetic dispersive analyzers.

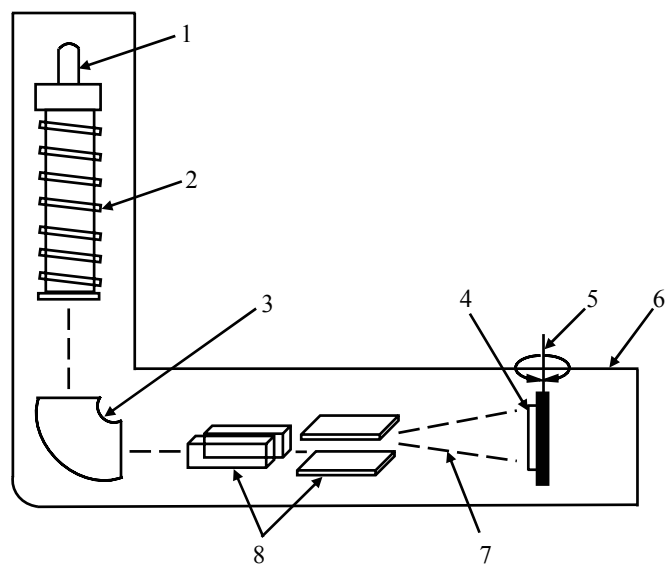

Fig. 1. The setup scheme for ion doping: 1 - source of ions, 2 accelerator of ions, 3 - magnet-analyzer, 4 - sample, 5sample rotation system, 6 - vacuum chamber, 7 - scanning ion beam, 8 - rejecting wafers.
As far as studied epitaxial silicon wafers were of (111)-oriented surface plane, chemical selective Sirtle etchant was used for level-by-level etching and revealing the defects. This etchant is intended for etching closepacked planes, and its chemical composition was as follows: $50 \mathrm{~g} \mathrm{CrO}_{3}+100 \mathrm{ml} \mathrm{H}$ Etching time was from 20 till $180 \mathrm{~s}$, etching speed was about $2-3 \mu \mathrm{m} / \mathrm{min}$. Dislocation etching poles on a surface, dislocation grid, sliding lines, separate dislocations, and small blocks of disordered silicon were among revealed defects.

\section{Experimental results}

Fig. 2 represents a typical overall defect picture on the surface of initial silicon HRESS wafers obtained by means of the electron scanning microscope "Cam Scan" after the process of chemical selective etching the wafers with Sirtle etchant within $2 \mathrm{~min}$. The observed triangular etching poles correspond to the presence of $60^{\circ}$ dislocations in the samples. After ion boron doping of HRESS wafers (ion beam energy was $100 \mathrm{keV}$, drive-in time was $12 \mathrm{~h}$ ) two dopant concentration maxima were observed in epitaxial layers by using the method of reverse voltage-capacitance characteristics (Fig. 3). This figure was obtained by means of an automatic voltagecapacitance curve tracer connected to a plotter.

The first maximum (nearest to the surface) is typical for the doping process (predepositing). If the annealing time of doped wafers (at the subsequent drive-in of boron impurity) is not enough, the dopant concentration maximum does not disappear completely, but only partially. The latter case is realised for the investigated samples. As far as the epitaxial layer doping was carried out by the method of ion implantation, the impurity concentration maximum is not located at the wafer surface, like to the case of usual diffusion, but at some distance from it [7]. The depth of the first maximum location in Fig. 3 is caused by the dopant atom free path,

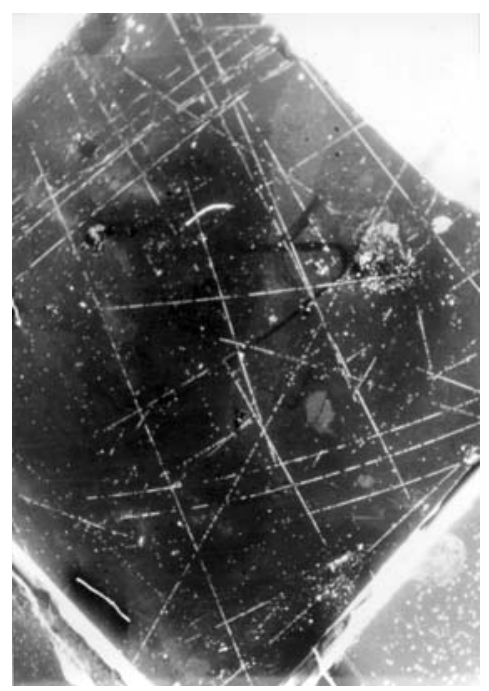

Fig. 2. The general view of dislocatins on HRESS (111) surface after selective chemical etching by Sirtle for $3 \mathrm{~min}$. Image is 2300 times increased. 


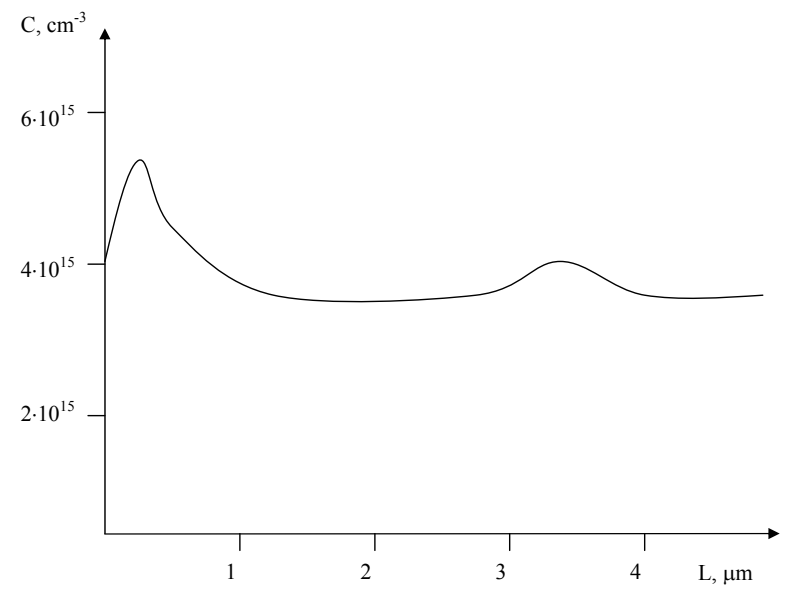

Fig. 3. Calculation results of boron concentration distribution along the depth of epitaxial wafers obtained by means of the voltage-capacitance curve tracer connected to a tablet curve-drawing instrument.

which, in its turn, depends on impurity and energy dose, and does not depend on structure perfection of doped samples. Level-by-level selective chemical etching of epitaxial silicon was used for studying defects of silicon crystal lattice in the field of the first maximum with the following research of the obtained surface by means of "PЭМ-106M". Knowing the speed of chemical etching, it was possible to control the distance from the silicon wafer surface. A strongly disordered (close to polycrystalline) silicon area (Fig. 4) has been found out at the depth $0.3 \mu \mathrm{m}$ from the wafer surface. For detailed studying the silicon heterogeneity observed at the depth $0.3 \mu \mathrm{m}$ (see Fig. 4) and corresponding to the position of the first boron concentration maximum (see Fig. 3), more careful surface research by means of AFM was carried out. This research allows to obtain the three-dimensional image of an interested area with the maximum resolution. Results of research are presented in Fig. 5. As obvious from the figure 5a, in the position of the first peak of boron concentration, a fine-blocked silicon structure is observed. To determine block sizes, the labels "1" and " 2 " were put and the straight line 1-2 was spent. Fig. $5 \mathrm{~b}$ shows sizes of blocks along this straight line, which confirms the existence of fine-blocked area in the investigated silicon structures.

SEMS studying the wafer surfaces by using "Cam Scan" and level-by-level chemical etching of epitaxial layers were carried out to research the nature of the second maximum. At the depth of 3-4 $\mu \mathrm{m}$ (for different samples it differs), dislocation grids were found out. Fig. 6 illustrates the typical pattern of a dislocation grid. The disorder in the depth position of dislocation grids is caused by the defective structure of epitaxial heterogeneity that varies not only from one sample to another but also within the same sample. Therefore, for some samples the dislocation grid is settled down not strictly parallel to a wafer plane, but at a small angle to it. Fig. 6 shows that the period of dislocation grid is changeable. The assumption was done that the

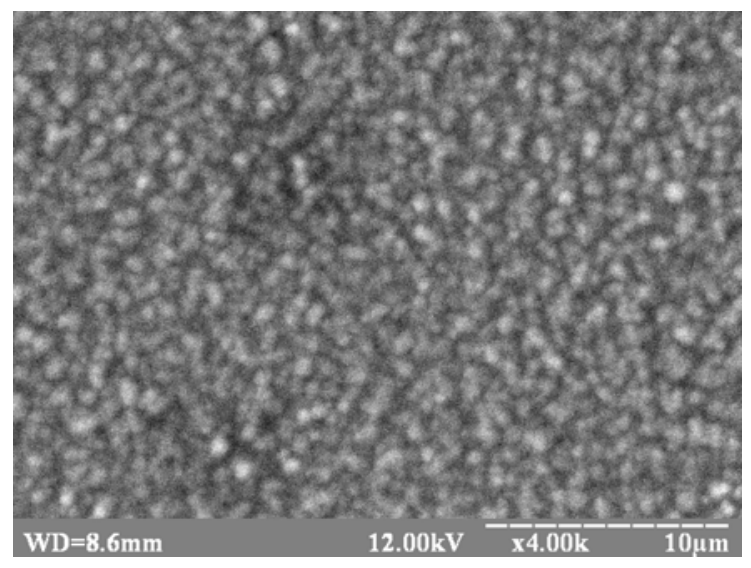

Fig. 4. SEMS image of B-doped silicon surface at the depth of $0.3 \mu \mathrm{m}$ from the wafer surface after selective chemical etching by Sirtle. Image is 4000 times increased.

distinction of the grid period is related with initial $i$-layer defects. SEMS comparison of HRESS and of ion doped epitaxial wafers obtained after ion doping of these HRESS shows that positions of dislocation areas in HRESS correspond to areas with the small period of grid in the samples (see Fig. 2).

\section{Discussion}

Epitaxial technology is always related with preparation of layers with various types of conductivity or doped at various levels and differing by properties both of the substrate and each other [12]. The mismatch of lattices defining relative deformation is

$\varepsilon^{\prime}=\omega \cdot C$,

where $C$ is the concentration; $\omega-$ Vegard coefficient that characterizes changes of the lattice parameter at doping. In the case of B-doped silicon, $\omega=2.8 \cdot 10^{-3}$ [13].

In the case when several kinds of impurity are present, a resultant relative deformation is defined as a sum of contributions:

$\varepsilon=\sum_{i} \varepsilon_{i}$

Calculation data of X-ray spectra for elements on the surface of a silicon wafer in the area of dislocation grids (aluminum layer was deposited to calibrate calculation results), as well as the boron concentration data obtained on the basis of voltage-capacitance curve tracer (see Fig. 3) are listed in Table. Apparently, oxygen atoms are the main uncontrollable (unlike boron) impurity in silicon.

To establish the contribution of oxygen and boron atoms in the value of relative deformations inherent to silicon crystal lattice (2) in the region of a dislocation grid, we have made calculations of each impurity element contribution according to the Vegard law (1). Results of calculations are given in Table.

\section{(C) 2009, V. Lashkaryov Institute of Semiconductor Physics, National Academy of Sciences of Ukraine}


Table. Values of stresses and relative deformations calculated for the investigated epitaxial silicon wafers.

\begin{tabular}{|l|l|l|l|l|}
\hline $\begin{array}{l}\text { Ele- } \\
\text { ment }\end{array}$ & $\begin{array}{l}\text { Atomic } \\
\text { percent }\end{array}$ & $\begin{array}{l}\text { Vegard } \\
\text { coefficient, } \\
\omega\end{array}$ & $\begin{array}{l}\text { Relative } \\
\text { deforma- } \\
\text { tion of } \\
\text { crystalline } \\
\text { lattice, } \\
\varepsilon(1)\end{array}$ & $\begin{array}{l}\text { Deforma- } \\
\text { tion } \\
\text { correspon- } \\
\text { ding upper } \\
\text { fluidity limit } \\
\text { (UFL), } \varepsilon_{U F L}\end{array}$ \\
\hline $\mathrm{Na}$ & 0.094 & & & \\
\hline $\mathrm{Si}$ & 93.812 & & & $1 \cdot 10^{-6}[14]$ \\
\hline $\mathrm{O}$ & 5.656 & $1 \cdot 10^{-4}[15]$ & $5.656 \cdot 10^{-4}$ & \\
\hline $\mathrm{Al}$ & 0.438 & & & \\
\hline Totals & 100.000 & & & \\
\hline $\mathrm{B}$ & 0.000008 & $\begin{array}{l}2.8 \cdot 10^{-3} \\
{[13]}\end{array}$ & $2.24 \cdot 10^{-8}$ & \\
\hline
\end{tabular}
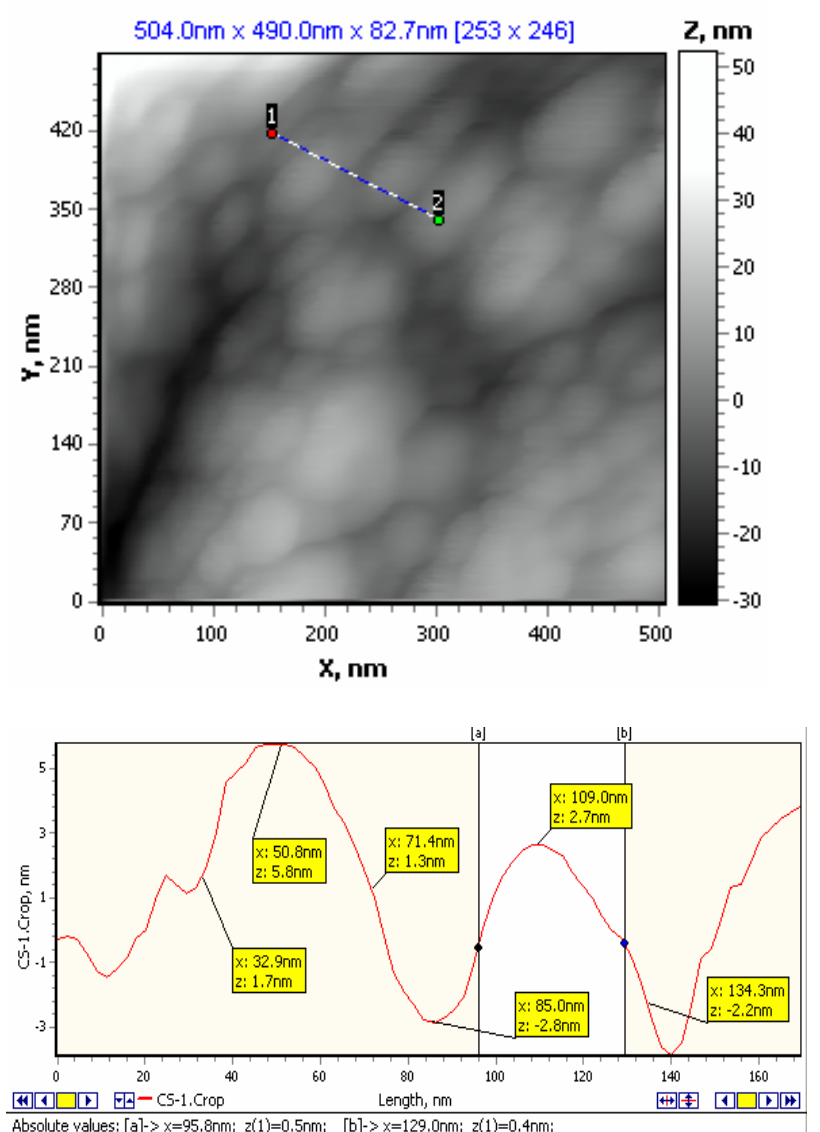

Absolute values: [a] $>x=95.8 \mathrm{~nm} ; z(1)=0.5 \mathrm{~nm} ; \quad[\mathrm{b}]->x=129.0 \mathrm{~nm} ; z(1)=0.4 \mathrm{~nm} ;$

Difference between markers: $\mathrm{dx}=33.3 \mathrm{~nm} ; \mathrm{dz}(1)=0.2 \mathrm{~nm}$;

Difference between first two lines: $x[a]=95.8 \mathrm{~nm}, \mathrm{dz}[\mathrm{a}]=0.0 \mathrm{~nm} ; \mathrm{x}[\mathrm{b}]=129.0 \mathrm{~nm}, \mathrm{dz}[\mathrm{b}]=0.0 \mathrm{~nm}$

Fig. 5. AFM image of silicon surface in the region of the first peak of dopant concentration: (a) - lateral image of silicon surface in the field of disordered layer (the straight line 1-2 is spent perpendicularly to edges of blocks to determine block sizes); (b) - sizes of blocks along the straight line 1-2 (abscissa is directed along the straight line $1-2$ and allows to determine the block width; the axis of ordinates is located in the figure plane and allows to determine the size of blocks along their length).
The analysis of the experimental data and calculations (see Table) has shown that the concentration of oxygen atoms in the region of dislocation grid is six orders higher than that of boron atoms. Therefore, deformations $\varepsilon_{\mathrm{O}}$ caused by the presence of oxygen atoms are four orders higher than the deformations $\varepsilon_{\mathrm{B}}$ caused by presence of boron atoms. Hence, it is possible to neglect $\varepsilon_{\mathrm{B}}$ in the expression (2) and consider the value of relative deformations in silicon crystal lattice within the region of dislocation grids equal to $\varepsilon_{0}$. It is necessary to underline that in this case we deal with residual (after annealing at $600{ }^{\circ} \mathrm{C}$ ) deformations of the crystal lattice in the region of dislocation grids and, accordingly, with the residual boron dopant concentration. For this reason, the structural defects in the form of disordered silicon and dislocation grids are observed in the positions of maxima in Fig. 3 where the boron concentration is lower than the threshold one. Actually, to form this defective structure the primary (before annealing process) boron concentration in these areas should be high enough. If in formula (1) we substitute the values of threshold deformations for pure silicon and of the Vegard coefficient for boron taken from Table, we will obtain that the boron concentration in the positions of both peaks before annealing was not less than $4.64 \cdot 10^{-4}$ atomic percents. Indeed, it is a very rough estimate, because threshold values are taken for pure instead of doped silicon containing, in addition, foreign impurities (oxygen) and initial structural defects (dislocation grid period is different owing to the influence of initial defects). There is an assumption [14] that atoms of oxygen raise silicon threshold of plasticity, therefore it is difficult to estimate the real value of upper fluidity limit (UFL) for these samples.

It is necessary to compare the values of $\varepsilon_{\mathrm{B}}$ and $\varepsilon_{\mathrm{O}}$ with that of $\varepsilon_{\mathrm{UFL}}$ for deformations corresponding to UFL of silicon, i.e., to the beginning of plastic flow in pure silicon.

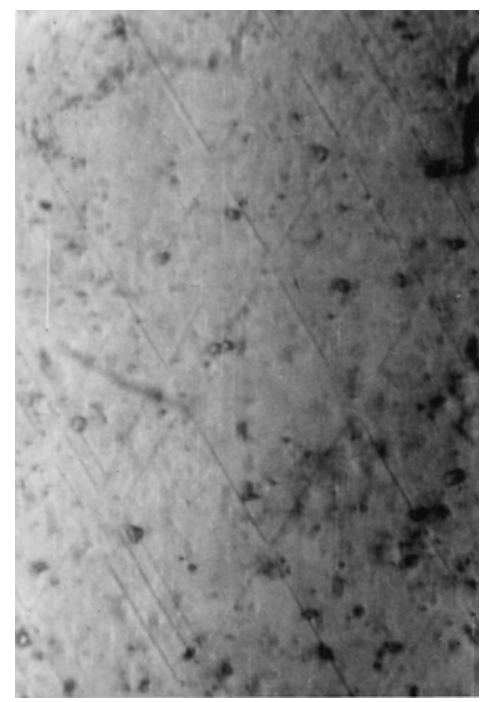

Fig. 6. Image of the oxygen precipitated dislocation grid in $p$ silicon obtained after selective chemical etching by Sirtle (depth of analysis $L=3.5 \mu \mathrm{m}$, image increase is 2300 times, (111) surface). 
As far as $\varepsilon_{\mathrm{B}}<<\varepsilon_{\mathrm{UFL}}$ and $\varepsilon_{\mathrm{O}} / \varepsilon_{\mathrm{UFL}}=435$ it is possible to assume that the existence of the second concentration maximum for boron impurity (lower in its height) is not the reason for dislocation grid formation at the depth of 3-4 $\mu \mathrm{m}$ from the wafer surface. Besides, in the region of the second maximum fine-blocked silicon similar to that of the first maximum was not revealed. However, it is not so: before doping there were initial defects in this crystal in the form of individual dislocations typical to this area. The dislocation grids were formed after ion doping. Besides, $\varepsilon_{\mathrm{B}}$ is calculated for the residual boron concentration (i.e. after annealing), but dislocation grid was formed after ion doping, that is, before annealing. The mentioned facts justify that the second maximum existence is caused by initial defects of epitaxial silicon.

Defects found in HRESS and stretchings of silicon crystal lattice caused by them in the process of ion implantation have served as a drain area for dopant and resulted in formation of the second maximum. Initial dislocations in HRESS at the depth of 3-4 $\mu \mathrm{m}$ already justify that in this area of a wafer there were the mechanical stretchings exceeding the threshold of plasticity. With existence of uncontrollable aggregation of oxygen atoms in the process of epitaxial layer growth with deformation of silicon crystal lattice caused by it, which 435 times exceeds the maximum of the allowed elastic deformation value for pure silicon, we relate the formation of dislocations in HRESS. With formation of dislocations, stress values became lower than the threshold ones (dislocations are accumulators of mechanical stresses), but never the same remained. When boron got into a stretched area, stresses have exceeded again a plasticity threshold owing to what dislocations were repeatedly formed.

It is necessary to notice that implantation of the same impurity can promote both stretching and compression of crystal lattice. It is defined by where the impurity is placed (either in the lattice sites, or interstitially), and also by what stress (negative, positive or zero) exists in the initial lattice.

So, interstitial (both boron and oxygen) impurity always promotes lattice stretching in a place of implantion without dependence on the covalent radius of impurity [16-19]. When boron impurity gets to a lattice site, lattice compression is observed, because the covalent radius of boron is smaller than that of silicon, and a negative change of lattice period occurs. On the contrary, when oxygen atom is in a lattice site, lattice stretching is observed, because oxygen possesses a larger covalent radius in comparison with that of silicon [16-19].

As far as dislocations, being accumulators of mechanical stresses, form stretching and compression areas, it allows to assume that one and the same interstitial atom, that in usual conditions always leads to a lattice stretching brought to the area of compression around dislocation, can lead to insignificant positive deformation (thus a resultant lattice deformation still will be negative), to a zero resultant deformation (that is to neutralise negative deformation), to increase of a lattice period. Realisation of every above-mentioned case is defined by initial deformation of a crystal, and also by the value of positive deformation brought by interstitial impurity atom. The latter, in its turn, is defined by the atomic structure and impurity concentration.

Similar conclusions can be made concerning the corresponding impurity atoms in a lattice site of a crystal possessing initial positive and negative deformations.

\section{Conclusion}

1. Calculation data of X-ray spectra for elements on surface of a silicon wafer in the area of dislocation grids showed that oxygen atoms are the main uncontrollable impurity in silicon.

2. It is established that the existence of the first dopant concentration maximum is caused by a small annealing time of doped wafers after subsequent drive-in for boron impurity. The existence of the second dopant concentration maximum is caused by initial defects of epitaxial silicon.

3. The variable period of a dislocation grid is related with the presence of unevenly distributed defects in initial silicon.

\section{References}

1. S.S. Kapur, M. Prasad, J.C. Crocker, T. Sinno, Role of configurational entropy in the thermodynamics of clusters of point defects in crystalline solids // Phys. Rev. B 72, 014119 (2005).

2. G. Davies, Sh. Hayama, L. Murin, R. KrauseRehberg, V. Bondarenko, A. Sengupta, C. Davia, A. Karpenko, Radiation damage in silicon exposed to high-energy protons // Phys. Rev. B 73, 165202 (2006).

3. H. Bracht, Self- and foreign-atom diffusion in semiconductor isotope heterostructures. I. Continuum theoretical calculations // Phys. Rev. B 75, 035210 (2007).

4. H. Bracht, H.H. Silvestri, I.D. Sharp, E.E. Haller, Self- and foreign-atom diffusion in semiconductor isotope heterostructures. II. Experimental results for silicon // Phys. Rev. B 75, 035211 (2007).

5. N.E.B. Cowern, Diffusion in a single crystal within a stressed environment // Phys. Rev. Lett. 99, 155903 (2007).

6. F.F. Komarov, O.I. Velichko, V.A. Dobrushkin, A.M. Mironov, Mechanisms of arsenic clustering in silicon // Phys. Rev. B 74, 035205 (2006).

7. K. Reivy, Defects and Impurities in Semiconductor Silicon. Mir, Moscow, 1984 (in Russian).

8. C.L. Allerd, X. Yuan, M.Z. Bazant, L.W. Hobbs, Elastic constants of defected and amorphous silicon with the environment-dependent interatomic potential // Phys. Rev. B 70134113 (2004). 
9. B.V. Petukhov, The effect of dynamic ageing of dislocations on deformation behaviour of semiconductors with impurities // Fizika Tekhnika Poluprovnikov 36 (2), p. 129-133 (2002) (in Russian).

10. E. Sirtle, A. Adler, Chromsaure-Flussaure als specifisches System zur Atzgrubenentwicklung auf Silizium // Z. Metallik 52 (8), S. 529-531 (1961).

11. J. Philibert, G. Fontaine, E. Vicario et al., Microanalysis and Scanning Electron Microscopy. Metallurgiya, Moscow, 1985 (in Russian).

12. L.N. Aleksandrov, Transitional Regions of Epitaxial Films in Semiconductors. Nauka, Novosibirsk, 1978 (in Russian).

13. F. Hubbard Horn, Densitometric and electrical investigation of boron in silicon // Phys. Rev. 97 (6), p. 1521-1525 (1955).

14. Yu.A. Kontsevoi, Yu.M. Litvinov, E.A. Fattakhov, Plasticity and Strength of Semiconductor Materials and Structures. Radio i svyaz', Moscow, 1982 (in Russian).
15. V.M. Babich, A.I. Bletskan, E.F. Venger, Oxygen in Monocrystals of Silicon. Interpress LTD, Kiev, 1997 (in Russian).

16. V.I. Plebanovich, A.I. Belous, A.R. Chelyadinskii, V.B. Odzhaev, Creation of dislocation-free iondoped silicon layers // Fizika Tverdogo Tela 50 (8), p. 1378-1382 (2008) (in Russian).

17. I.N. Smirnov, Changes of silicon lattice parameter caused by boron, arsenic and antimony diffusion // Doklady Akademii nauk SSSR. Tehnicheskaya fizika 221 (2), p. 332-334 (1975) (in Russian).

18. I.N. Smirnov, I.I. Petrov, T.F. Goryacheva, Research of boron diffusion in silicon by X-ray diffraction methods // Elektronnaya tehnika. Seriya 2 (Poluprovodnikovye pribory) 97 (5), p. 11 18 (1975) (in Russian).

19. I.N. Smirnov, Deformation of silicon lattice caused by bombardment with boron and oxygen ions // Doklady Akademii nauk SSSR. Fizicheskaya khimiya 225 (3), p. 621-623 (1975) (in Russian). 